\title{
Feature Analysis on Atmosphere Laser Communication Channel Under Rainy Condition in the Tibetan Plateau
}

\author{
Wei Xiao*,1,2 \\ ${ }^{I}$ Electronic Information School, Wuhan University, Wuhan, Hubei, 430072, P.R. China \\ ${ }^{2}$ School of Engineering, Tibet University, Lhasa, Tibet, 850000, P.R. China
}

\begin{abstract}
In the Tibetan Plateau, due to lack of raw experimental data sets and proper data analysis method, investigations on atmosphere laser communication channel (ALCC), especially under rainy condition, are rarely concerned by researchers. Neural network group and optimal weight initialization technology (OWIT) are adopted in the analysis process. Firstly, construct neural network group according to different season's conditions. Secondly, utilize existed raw data sets of ALCC under rainy condition to choose matching initial weight sets with OWIT. Thirdly, train neural network group until expected requirement is met. Finally, load rain data sets from the Tibetan Plateau (Lhasa for example) on trained neural network group to achieve the ultimate channel quality of ALCC. Actual results show that spring rain has the best quality of ALCC, followed by winter rain, summer rain and autumn rain.
\end{abstract}

Keywords: Atmosphere laser communication channel (ALCC), neural network group, optimal weight initialization technology(OWIT), the Tibetan Plateau.

\section{INTRODUCTION}

The Tibetan Plateau (called Qinghai-Tibet Plateau), also known as Himalayan Plateau, is a vast plateau covering most of the Tibet Autonomous Region and Qinghai Province in western China. Free-space optical communication (FSO) is an optical communication technology which adopts light propagating in free space to transmit data. FSO is a costeffective technique which has application prospects in the Tibetan Plateau.

"Free space" is often in visible distance, using air, outer space, vacuum, or something similar as transmission medium, which has great uncertainty compared with cable communication system. ALCC is a channel adopted atmosphere as transmission medium, which is very vulnerable for bad weather condition, such as fog, rain etc. [1].

Investigations of ALCC under rainy condition mainly are focused on tropical region [2-4], or heavy rain condition [5-7].

Rainfall mainly occurs from May to October in the Tibetan Plateau. And rain studies are concentrated on the mechanism $[8,9]$, which have no direct support to study on ALCC. In sum, the Tibetan Plateau is rarely concerned by researchers in this field.

*Address correspondence to this author at the Electronic Information School, Wuhan University, Wuhan, Hubei, 430072, P.R. China;

Tel: +86 13889084074; Fax: +862768775770.

E-mail: tibetxiaowei@qq.com

\section{THE MAIN DIFFICULTIES IN ANALYZING ALCC UNDER RAINY CONDITION IN TIBETAN PLATEAU}

At present, studies on ALCC in the Tibetan Plateau are at the beginning stage. Lack of direct raw experimental data sets is the main difficulty. To obtain direct raw experimental data sets, expensive measure equipments, professional technicians and other resources are fundamental conditions. However, they are too difficult to get.

Specific date, rainfall and rain intensity are available from China Meteorological Administration. Using the data sets above, how to achieve the quality of ALCC in Tibetan Plateau?

Available data sets and actual demand are described in Fig. (1). It is difficult to build direct mathematic model between available data sets and channel quality from Fig. (1). Naturally, traditional mathematic method is not applicable to the problem. Artificial intelligence technology seems more feasible to solve it.

\section{MAIN IDEA OF THE SOLUTION}

\subsection{Introduction of the Solution}

New solution, which only adopts meteorological data in the Tibetan Plateau, can get ultimate result of ALCC under rainy condition. The main idea of the solution can be divided into the following two steps.

Step1: Training neural network group with existed raw data sets of ALCC until all networks converge; 
Available weather data in

Qinghai-Tibet Plateau
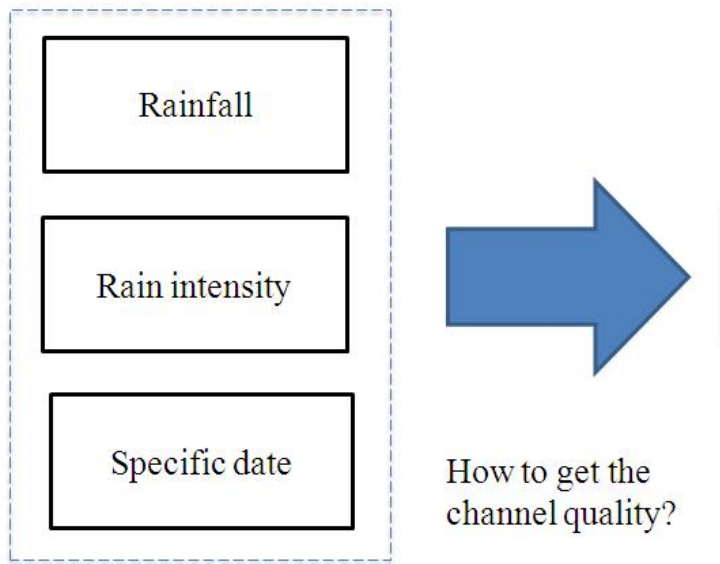

\section{Quality of ALCC}

under rain condition

\section{How to get the}

channel quality?

Fig.(1). Problem description.

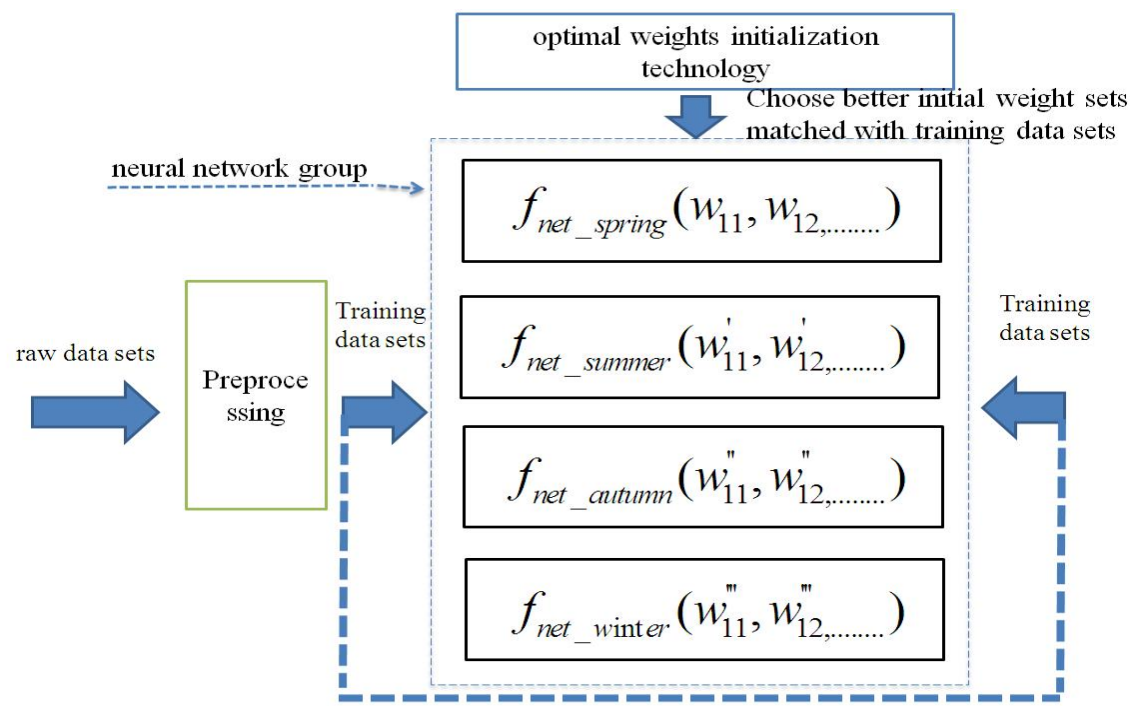

Fig.(2). Main idea of the solution.

Step 2: Adopting meteorological data in the Tibetan Plateau (Lhasa for example) to achieve ultimate result from trained neural network group.

Existed raw data sets of ALCC record specific date, rainfall, rain intensity and bit error rate (BER, which is quality of ALCC) [10]. In order to achieve better analysis, specific date is transferred into season. Therefore, neural network group is adopted, which can match different seasons. (Fig.2):

The main idea of the solution can be described as follows

1) Construct neural network group according to the size of existed raw data sets (multi-layer perceptron network(MLPN) is adopted in the project).

2) Using existed raw data sets of ALCC under rainy condition to choose matching weight sets with OWIT.
3) Training neural network group with existed raw data sets until expected requirement is met.

4) Achieve ultimate output result by using data sets from the Tibetan Plateau (Lhasa for example) to trained neural network group(Fig.3).

\subsection{Specific Steps of OWIT}

In (2), OWIT is adopted to assess the matching degree between the initial weight sets of MLPN and current training data sets (also called sample data sets), so that good initial weight sets can be achieved in the neural network. Fig. (4) is the flowchart of OWIT.

Random number sets are generated by weight sets generator, which is loaded on MLPN. Then the matching degree between weight sets and current training data sets is calculated 


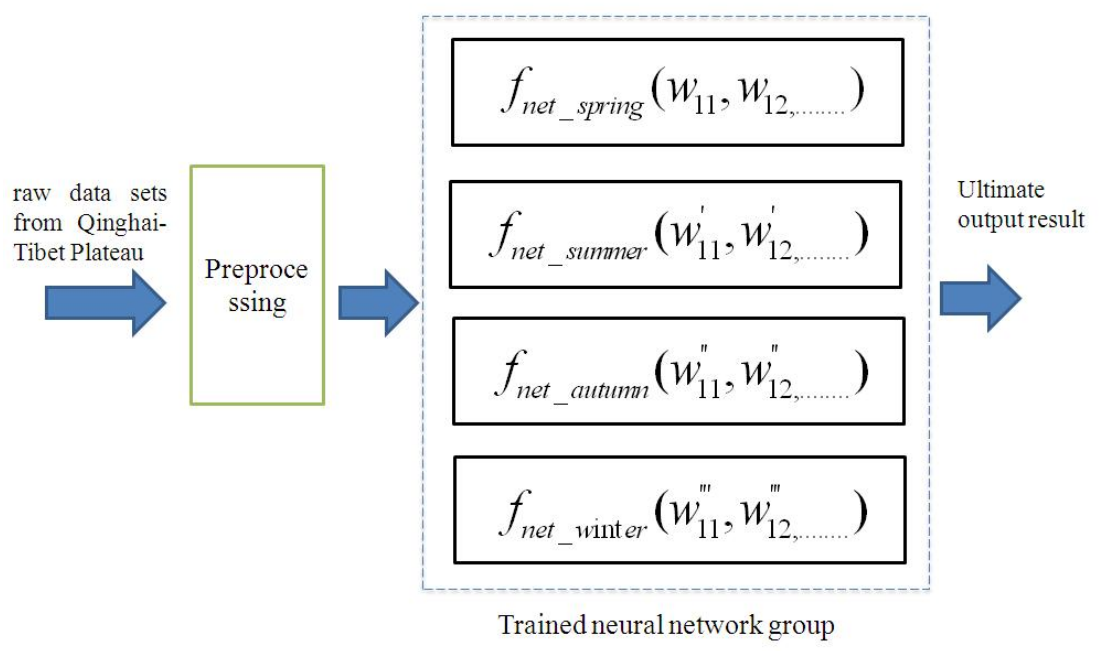

Fig. (3). Achieving ultimate result from trained network group.

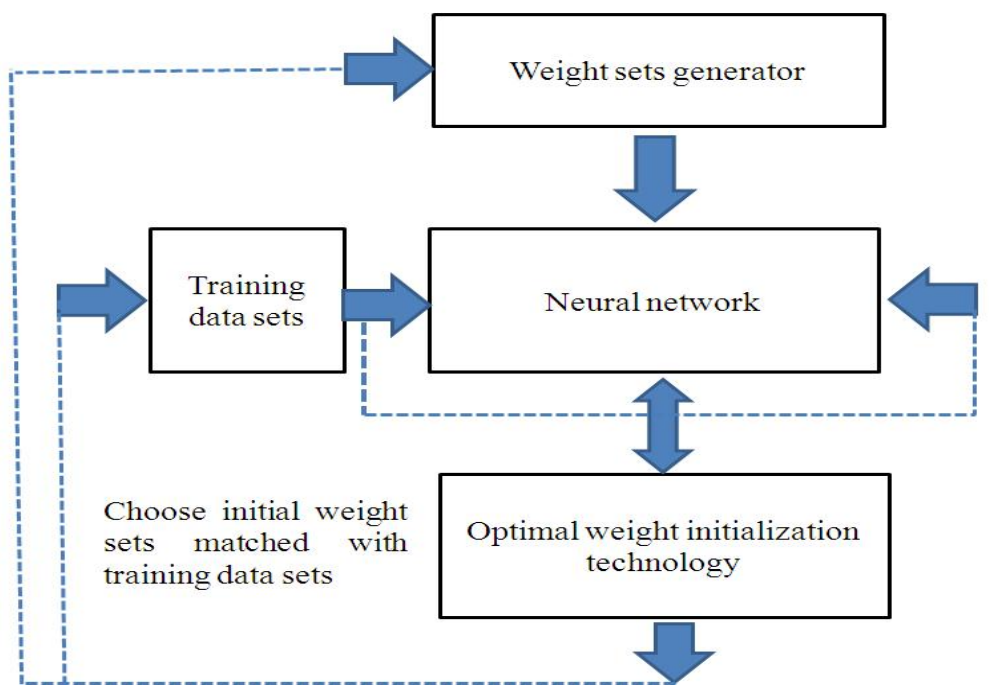

Fig. (4). The flowchart of OWIT.

by means of OWIT. Consequently, the weight sets which has better matching degree will be kept in MLPN[11].

The core of OWIT can be described by Equations (1)-(3).

$J_{i^{\prime}}(w)=\sum_{i^{\prime}=1}^{Q}\left(\sum_{i=1}^{M} \sum_{k=1}^{H^{\prime}} J_{i}^{k}(w)+\sum_{k=1}^{H^{\prime}} J^{k}(w)\right) /\left(N^{*} Q\right)$

$Q$ is the number of sample data sets of MLPN. $M$ is the number of neuron in current layer, and $H^{\prime}$ is layer number. $N$ is the number of weights. The term on the left-hand of Equation (1) is the average weight discrete degree in MLPN.

Total error of MLPN is defined by Equation (2).

$$
E(w)=\sqrt{\frac{1}{Q} \sum_{i^{\prime}=1}^{Q} \sum_{i=1}^{R}\left(t_{i^{\prime} i}-z_{i^{\prime} i}\right)^{2}}
$$

$R$ is the number of output neuron in MLPN. The vector $t$ is target response included in sample data sets, and vector $z$ is the actual result of MLPN.

Combined with weight discrete degree and total error of MLPN, Equation (3) is achieved to estimate matching degree between current weight sets and current sample data sets.

$$
\begin{aligned}
& L(w)=\frac{\sqrt{N}}{E(w)}+\left[\sum_{i=1}^{Q}\left(\sum_{i=1}^{M} \sum_{k=1}^{H^{\prime}} J_{i}^{k}(w)+\sum_{k=1}^{H^{\prime}} J^{k}(w)\right) /\right. \\
& \left.\left(N^{*} Q\right)-\sqrt{N}\right]^{-1}
\end{aligned}
$$

$L(w)$ is the ultimate optimization criteria function to quantify the value of matching degree. The first term on the right-hand estimates the convergence degree of MLPN. The second term on the right-hand presents discrete degree of current weight sets[11]. 




Fig. (5). Rainfall distribution graph from 1997 to 2006.

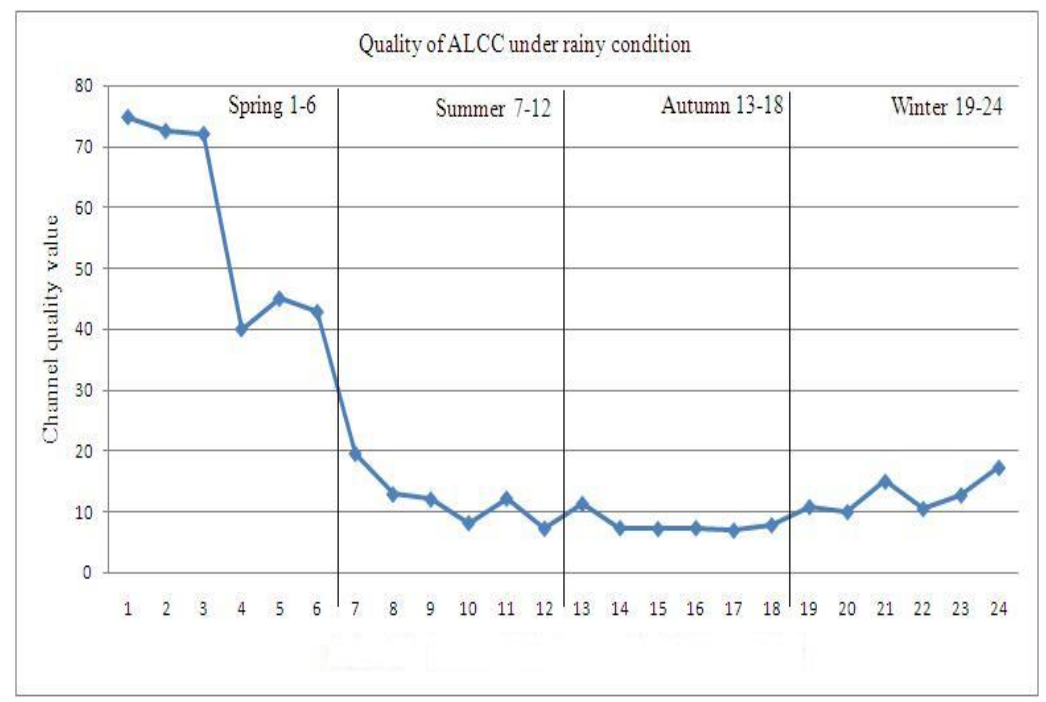

Fig, (6). Channel quality value in different seasons in the Tibet Plateau (Lhasa for example).

\section{ACHIEVEMENT OF FEATURE ANALYSIS}

\subsection{Introduction of Raw Rainy Weather Data in the Ti- betan Plateau (Lhasa for Example)}

Fig. (5) is the rainfall distribution graph from 1997 to 2006 in Lhasa. From the rainfall graph above, the rain weather mainly occurs from May to October, where heavy rain and violent rain have greater possibility in this period. Thus, rain has evident seasonal feature in the Tibet Plateau.

\subsection{Existed Data Sets of ALCC Under Rainy Condition}

Table 1 records existed raw experimental data sets of ALCC under rainy condition. Specific date, rain intensity, rainfall and BER are recorded in the data sets[10]. And BER represents the quality of ALCC.

\subsection{Preprocessing of Existed Data Sets of ALCC Under Rainy Condition}

Preprocessing is adopted to transform raw data sets into data sets which can be accepted by individual neural network. In Table 1, specific date is converted into season according to relationship between Solar Calendar and Han calendar (Table 2), which can further match with different neural networks. Rain intensity can be quantized according to rain type. Rainfall and BER can be quantized directly.

Table 2 shows the relationship between Solar Calendar and Han Calendar, which can further be transformed into season.

\section{CONCLUSION}

\subsection{Quality of ALCC Under Rainy Condition in the Ti- betan Plateau (Lhasa for Example)}

In Fig. (6), the horizontal axis is seasons, which sample rain weather from spring, summer, autumn and winter according to the time order respectively. The vertical axis is channel quality value, which is inversely proportional to BER. 
Table 1. Raw experimental data sets of ALCC under rainy condition(part of the data sets).

\begin{tabular}{|c|c|c|c|}
\hline $2007-7-4$ & Heavy rain & 19.8 & 6.8 \\
\hline $2007-6-17$ & Moderate rain & 27.5 & 3.5 \\
\hline $2007-8-9$ & Heavy rain & 28.6 & 7.7 \\
\hline $2007-8-31$ & Moderate to light rain & 2.4 & 3.8 \\
\hline $2007-9-3$ & Moderate to light rain & 10.1 & 3.7 \\
\hline $2007-10-8$ & Light rain rain(drizzle) & 0.2 & 0.76 \\
\hline $2007-10-27$ & Heavy rain & 20.3 & 7.5 \\
\hline $2007-5-13$ & Moderate rain & 13.6 & 5 \\
\hline $2007-3-15$ & Moderate rain & 10.3 & 3.3 \\
\hline $2007-5-23$ & Light to moderate rain & 9.2 & 4.7 \\
\hline $2007-5-24$ & Light rain(drizzle) & 0.8 & 1.5 \\
\hline
\end{tabular}

Table 2. The mapping table of Solar Calendar, Han Calendar and season.

\begin{tabular}{|c|c|c|}
\hline Solar Calendar & Han Calendar & Season \\
\hline $2007-2-18$ to $2007-3-18$ & $2007-1-1$ to $2007-1-29$ & Spring \\
\hline $2007-4-17$ to $2007-5-16$ & $2007-3-1$ to $2007-3-30$ & Spring \\
\hline $2007-6-15$ to $2007-7-13$ & $2007-5-1$ to $2007-5-29$ & Summer \\
\hline $2007-7-14$ to $2007-8-12$ & $2007-6-1$ to $2007-6-30$ & Summer \\
\hline $2007-8-13$ to $2007-9-10$ & $2007-7-1$ to $2007-7-29$ & Autumn \\
\hline $2007-9-11$ to $2007-10-10$ & $2007-8-1$ to $2007-8-30$ & Autumn \\
\hline $2007-12-10$ to $2008-1-7$ & $2007-11-1$ to $2007-11-29$ & Winter \\
\hline $2008-1-8$ to $2008-2-6$ & $2007-12-1$ to $2007-12-30$ & Winter \\
\hline
\end{tabular}


From Fig. (6), channel quality value has the highest value in spring, followed by winter, summer and autumn respectively. According to statistical data, heavy rainfall has greater possibility in summer and autumn, which further has bad impact on quality of ALCC. And light rain is likely to appear in spring, which has a little influence on free space optical communications. Thus, spring rain has the best quality of ALCC in the Tibet Plateau, followed by winter rain. Summer rain and autumn rain have the worst quality of ALCC.

\subsection{Summary and Prospect}

Several contributions are made in this paper.

1) Channel quality value in different seasons in the Tibetan Plateau is acquired successfully only through recorded meteorological data, which has important reference value for the further research on ALCC.

2) A workable architecture has been constructed to extract feature information from existed data sets and store it in neural network group, which can be adopted in similar applications.

3) Using OWIT to choose matching weight sets, can accelerate the convergence speed of MLPN [11].

Neural network group, combined with OWIT, not only can be used for analysis of ALCC, but also be adopted in other areas. It provides a good solution which is efficient for information acquisition inside the data sets.

\section{CONFLICT OF INTEREST}

The author confirms that this article content has no conflict of interest.

\section{ACKNOWLEDGEMENTS}

This work was financially supported by the National Natural Science Foundation of China (No. 61163013) and Aviation Science Foundation of China (No. 201351S5002).

\section{REFERENCES}

[1] A. Vavoulas, H.G. Sandalidis, and D. Varoutas, "Weather effects on FSO network connectivity", IEEE/OSA Journal of Optical Communications and Networking, vol. 4, no. 10, pp. 734-740, 2012.

[2] S.A. Al-Gailani, A.B. Mohammad, U.U. Sheikh, and R.Q. Shaddad, "Determination of rain attenuation parameters for free space optical link in tropical rain", International Journal for Light and Electron Optics, vol. 125, no. 4, pp. 1575-1578, 2014.

[3] S.A. Zabidi, M.R. Islam, W. Al-Khateeb, and A.W. Naji, "Investigating of rain attenuation impact on Free Space Optics propagation in tropical region", In: $4^{\text {th }}$ International Conference on Mechatronics, 2011, pp. 1-6.

[4] M. Yahya, K. Salleh, N.A. Mohd Akib, and Z. Awang, "Low cost hybrid RF-FSO to reduce rain effect in tropical region", IEEE International RF \& Microwave Conference, 2011, pp. 250-253.

[5] A.Z. Suriza, I.M. Rafiqul, A.K. Wajdi, and A.W. Naji, "Effects of rain intensity variation on rain attenuation prediction for Free Space Optics (FSO) links", In: International Conference on Computer and Communication Engineering, 2012, pp. 680-685.

[6] S.A. Al-Gailani, A.B. Mohammad, and R.Q. Shaddad, "Enhancement of free space optical link in heavy rain attenuation using multiple beam concept", Optik, vol. 124, no. 21, pp. 4798-4801, 2013.

[7] P. Singh, and M.L. Singh, "Experimental determination and comparison of rain attenuation in free space optic link operating at 532 $\mathrm{nm}$ and $655 \mathrm{~nm}$ wavelength", Optik, vol. 125 , no. 17, pp. 45994602, 2014.

[8] Y.F. Fu, and G.S. Liu, "Possible misidentification of rain type by TRMM PR over Tibetan plateau", Journal of Applied Meteorology and Climatology, vol. 46, no. 5, pp. 667-672, 2007.

[9] H. Yamada, and H. Uyeda, "Transition of the rainfall characteristics related to the moistening of the land surface over the central Tibetan Plateau during the summer of 1998", Monthly Weather Review, vol. 134, no. 11, pp. 3230-3247, 2006.

[10] X. He, and Z. Yin, Coding Theory in Wireless Laser Communication System, Science Press of China, 2009, pp. 245-246.

[11] X. Wei, and X.T. Yan, "The application of optimal weights initialization algorithm based on information amount in multi-layer perceptron networks", In: $3^{\text {rd }}$ IEEE International Conference on Computer Science and Information Technology, vol. 6, pp. 196-198, 2010.

\begin{tabular}{lll}
\hline Received: October 16, 2014 & Revised: December 23, 2014 & Accepted: December 31, 2014 \\
(C) Wei Xiao; Licensee Bentham Open. & &
\end{tabular}

This is an open access article licensed under the terms of the Creative Commons Attribution Non-Commercial License (http://creativecommons.org/licenses/bync/4.0/) which permits unrestricted, non-commercial use, distribution and reproduction in any medium, provided the work is properly cited. 